\title{
$F$ center in lithium fluoride revisited: Comparison of solid-state physics and quantum-chemistry approaches
}

\author{
Ferenc Karsai, ${ }^{1}$ Paul Tiwald, ${ }^{2}$ Robert Laskowski, ${ }^{3,1}$ Fabien Tran, ${ }^{1}$ David Koller, ${ }^{1}$ \\ Stefanie Gräfe, ${ }^{2,4}$ Joachim Burgdörfer, ${ }^{2}$ Ludger Wirtz, ${ }^{5,6}$ and Peter Blaha ${ }^{1}$ \\ ${ }^{1}$ Institute of Materials Chemistry, Vienna University of Technology, Getreidemarkt 9/165-TC, A-1060 Vienna, Austria, EU \\ ${ }^{2}$ Institute for Theoretical Physics, Vienna University of Technology, Wiedner Hauptstraße 8-10, A-1040 Vienna, Austria, EU \\ ${ }^{3}$ Institute of High Performance Computing, A*STAR 1 Fusionopolis Way, \# 16-16, Connexis, Singapore 138632 \\ ${ }^{4}$ Institute of Physical Chemistry and Abbe Center of Photonics, Friedrich-Schiller-University Jena, \\ Helmholtzweg 4, D-07743 Jena, Germany, EU \\ ${ }^{5}$ Physics and Materials Science Research Unit, University of Luxembourg, 162 a avenue de la Faïencerie, \\ L-1511 Luxembourg, Luxembourg, EU \\ ${ }^{6}$ Institute for Electronics, Microelectronics, and Nanotechnology (IEMN), CNRS UMR 8520, Dept. ISEN, \\ F-59652 Villeneuve d'Ascq Cedex, France, EU
}

(Received 20 December 2013; revised manuscript received 1 March 2014; published 26 March 2014)

\begin{abstract}
We revisit the theoretical description of the $F$ color center in lithium fluoride employing advanced complementary ab initio techniques. We compare the results from periodic supercell calculations involving density-functional theory (DFT) and post-DFT techniques with those from the embedded-cluster approach involving quantumchemical many-electron wave-function techniques. These alternative approaches yield results in good agreement with each other and with the experimental data provided that correlation effects are properly taken into account.
\end{abstract}

DOI: 10.1103/PhysRevB.89.125429

PACS number(s): 71.55.-i, 71.23.An, 71.15.-m

\section{INTRODUCTION}

Wide-gap insulators featuring color centers are considered in a growing number of optical applications [1], such as tunable solid-state lasers [2], and they have been investigated extensively [3-10] since the 1950s. Many different defects can be hosted by these materials, which are introduced by exposure of the crystal to high-energy photons, charged particles, or neutrons. The subject of this paper is the investigation of the $F$ center in lithium fluoride $(\mathrm{LiF})$, the simplest defect, where an electron is trapped at a vacant fluorine site. The host $\mathrm{LiF}$ is a prototypical wide-band-gap insulator with the largest known band gap [11] of $14.2 \mathrm{eV}$. Dawson and Pooley [12], Schwartz et al. [13], and Baldacchini et al. [14] observed the maximum of its experimental absorption peak at $5.08 \mathrm{eV}(T \approx$ $5 \mathrm{~K}$ ), at $4.98 \mathrm{eV}$ (room temperature), and at $5.07 \mathrm{eV}$ (room temperature) by optical absorption spectroscopy, respectively.

Several first-principles theoretical investigations for $F$ centers in LiF have been published in the past few decades, but none of them give results that are in close agreement with experiment. These calculations are either based on densityfunctional theory (DFT) $[15,16]$ treating the extended system using periodic boundary conditions, or on quantum-chemical methods [16-19] explicitly treating a cluster. Previous DFT calculations were based on the local-density approximation (LDA) suffering from the well known underestimate of the band gap for insulators [20-22] and the unphysical delocalization of localized states. Another problem in previous calculations is the use of the independent-particle approximation within which the electron-hole interaction is not properly accounted for in the determination of the excitation energies of the $F$ center. Previous quantum-chemical calculations were performed on the Hartree-Fock level neglecting correlation effects from the outset. Moreover, limited cluster and basis-set sizes restrict the accuracy of these calculations.
In the past few years, a few advanced periodic supercell calculations of defects have been performed with the methods of $a b$ initio many-body perturbation theory, such as the $G W$ approximations [23,24] and the Bethe-Salpeter equation [25,26] for other materials such as $\mathrm{CaF}_{2}$ [27], SiC [28], $\mathrm{BN}$ [29], $\mathrm{MgO}$ [30], and diamond [31]. However, to our knowledge, no such calculation has been performed for LiF. Moreover, no quantum-chemistry calculation of comparable sophistication appears available for a comparison.

The goal of the present paper is to provide a detailed comparison of different approaches, the "solid-state physicist's approach" ( $G W+$ Bethe-Salpeter for periodic supercells) and the "quantum chemist's approach" (correlated wave-function methods for embedded clusters). It is of interest to investigate the role of seemingly different correction terms within these complementary approaches for the $F$-center excitation spectrum and to check on their quantitative agreement.

The structure of this paper is as follows: In Sec. II, we present an overview of and comparison between these alternative approaches. Details of the solid-state physics approach and a comparison of different levels of approximation will be given in Sec. III. In particular, we will compare the performance of hybrid-DFT using the Yukawa-screened hybrid functional YS-PBE0 [32] and the TB-mBJ [33] potential with the results of $G W$ calculations. In Sec. IV, we will introduce the quantumchemistry methods employed within the present embeddedcluster approach (ECA). A detailed comparison between the results of different methods and with the experiment will be given in Sec. V.

\section{OVERVIEW OVER THE ALTERNATIVE APPROACHES}

On an intuitive level, the physics of the $F$ center in LiF, i.e., an electron trapped in an $\mathrm{F}^{-}$vacancy, can be thought of as a particle in a three-dimensional box [34,35], with a 
I. Extended-system approach (many-body perturbation theory) (a) DFT

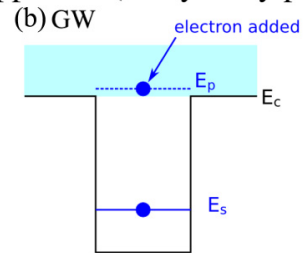
(c) Bethe-Salpeter
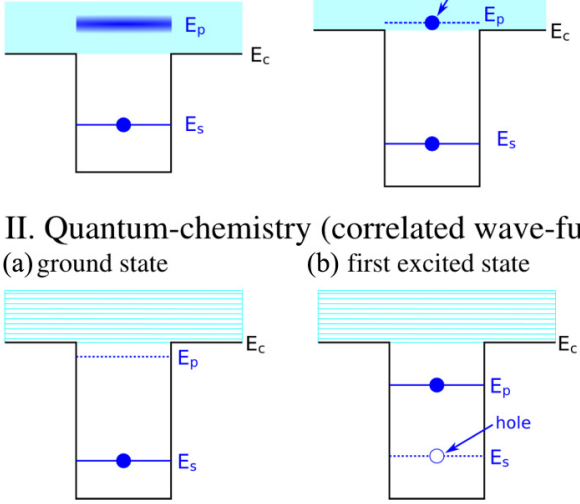

II. Quantum-chemistry (correlated wave-function) approach (a) ground state

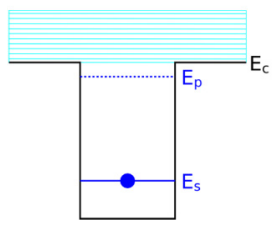

FIG. 1. (Color online) Alternative strategies for calculating $F$-center excitation energies. The $F$-center electron is represented by a particle in the box. $E_{s}$ denotes the single-particle level of the electron in the ground state ("s-like wave function") of the $F$ center, and $E_{p}$ denotes the energy of the first excited state (" $p$-like wave function"). The shaded area marks the conduction band with the band edge at $E_{c}$. In the extended system approach (I) of solid-state physics, the addition energy is determined by the $G W$ approximation (Ib), and the correction due to the excitonic interaction with the hole (Ic) is described by the Bethe-Salpeter equation. In embedded-cluster approaches employing quantum chemistry, the total $N$-electron energy of the two lowest states of opposite parity (IIa and IIb) are calculated and subtracted from each other.

width approximately given by the lattice constant. Thus, the $F$ center of $\mathrm{LiF}$ represents one of the simplest examples of a localized defect and its excitation. For this system, an embedded-cluster approach of modest size can be compared with methods describing defects and excitations within the framework of supercell methods treating extended systems in the solid-state context.

The alternative points of departure of these complementary approaches are schematically depicted in Fig. 1. For simplicity, the approximate "box" potential of this quasi-one-electron problem is illustrated as a square-well potential of finite height. The upper edge of the square well marks the onset of the conduction band, $E_{c}$. The ground-state energy of the electron is called $E_{s}$ in analogy to a hydrogenic $s$ state since the one-electron wave function is nodeless and nearly isotropic. It corresponds to a defect state deep in the band gap of LiF. The first excited state has a nodal plane and is triply degenerate, resembling the hydrogen $p$-state even though the problem at hand is evidently more complex. Since the square well has a finite depth, the $p$-state does not necessarily lie below the continuum onset, i.e., the conduction-band edge, but it may appear as a resonance within the conduction band. This is particularly true for calculations performed on the level of DFT, which is known to underestimate band gaps and therefore also underestimates the depth of the square-well potential. This scenario is depicted in panel I (a), where the $p$-state appears as a broad unoccupied band of resonances within the conduction band.

In calculations of extended systems, the underestimate of the band gap is usually corrected by employing many-body perturbation theory on the level of the $G W$ approximation
[23,36,37]. The resulting quasiparticle energies correspond to electron addition and removal energies. In the present case [panel I (b)], the quasiparticle energy yields the energy of an electron in the $p$-state in addition to an electron in the $s$-state, or, drawing on the hydrogenic analog, the energy of the $\mathrm{H}^{-}$ $(1 s, 2 p)$ resonance. While the depth of the potential well has increased considerably, the $p$-state is still in the conduction band due to the mutual repulsion with the electron in the $s$ state. However, it has been considerably localized, as indicated by the narrowing of the energy level. Since optical absorption corresponds to an excitation of a defect that preserves charge neutrality, a further correction is called for to account for the fact that the $s$-state is now unoccupied and the added $p$ electron interacts via screened Coulomb interactions with the hole. This gives rise to an excitonic state that may (or may not) lie below the edge of the conduction band [panel I (c)]. These excitonic effects can be calculated on the level of the Bethe-Salpeter equation $[25,26,37,38]$.

The quantum-chemistry approach to optical excitation energies is conceptually complementary to the approach from solid-state physics. Rather than successively improving on the mean-field independent-particle energies and switching on the electron-hole interaction at the end, one attempts to calculate the ground-state and excited-state many-body wave functions and the corresponding total energies directly by placing the electron in the $s$-state or the $p$-state [see panel II (a) and (b)]. The assignment and occupation of the different many-electron defect states can easily be controlled as the $s$ - and $p$-states correspond to the energetically lowest state of a given symmetry, in the present case with opposite parity. The excitation energy is then the difference between total energies of the $N$-electron states. Excitonic effects are thus naturally included from the outset. Typically, the starting point of quantumchemistry calculations is the Hartree-Fock approximation. This approximation usually overestimates band gaps and thus also the depth of the potential well. Therefore, both the $s$ - and $p$-orbital energies are below the continuum onset. In turn, one improves on the Hartree-Fock energy difference by calculating ground and excited states accounting for correlation effects by using more sophisticated quantum-chemistry methods. We will show below that second-order perturbation theory based on a single restricted open-shell Hartree-Fock determinant is sufficient to reach accurate $F$-center excitation energies.

We note parenthetically that the $G W+$ Bethe-Salpeter approach is not necessarily restricted to calculations in periodic supercells. With a proper dielectric embedding, the methodology would be, in principle, applicable to embedded clusters. Conversely, correlated wave-function methods can also be implemented for periodic systems [39]. However, due to the unfavorable scaling with systems size, application to large systems is still very rare.

\section{THE SOLID-STATE-PHYSICS APPROACH}

\section{A. Relaxed geometry}

The calculations in the periodic supercell approach are based on DFT calculations performed with the code WIEN2K [40]. We use radii of 1.57 and $2.02 \mathrm{Bohr}$ for lithium and fluorine, respectively, and an energy cutoff parameter $\left(R K^{\mathrm{max}}\right)$ 
TABLE I. PBE vacancy-formation energies for the reaction $\mathrm{Li}_{x} \mathrm{~F}_{x} \rightarrow \mathrm{Li}_{x} \mathrm{~F}_{x-1}+\mathrm{F}$ and lattice relaxation parameters as a function of unit-cell (UC) size and the underlying lattice type (fcc: face-centeredcubic, bcc: body-centered-cubic, sc: simple-cubic). $E_{\text {rem }}$ : removal energies for a single fluorine atom without structural relaxations, $E_{\text {rel }}$ : relaxation energies, $d_{\mathrm{Li}}, d_{\mathrm{F}}$ : relaxation distances away from the vacancy of nearest-neighbor lithium and fluorine atoms. All energies in $\mathrm{eV}$, distances in $\AA$.

\begin{tabular}{lccccc}
\hline \hline $\mathrm{UC}$ & Lattice & $E_{\text {rem }}$ & $E_{\text {rel }}$ & $d_{\mathrm{Li}}$ & $d_{\mathrm{F}}$ \\
\hline $\mathrm{Li}_{8} \mathrm{~F}_{7}$ & fcc & 8.209 & -0.010 & 0.02 & 0.00 \\
$\mathrm{Li}_{16} \mathrm{~F}_{15}$ & $\mathrm{bcc}$ & 8.208 & -0.014 & 0.04 & 0.01 \\
$\mathrm{Li}_{32} \mathrm{~F}_{31}$ & $\mathrm{sc}$ & 8.213 & -0.015 & 0.04 & 0.01 \\
$\mathrm{Li}_{64} \mathrm{~F}_{63}$ & fcc & 8.221 & -0.014 & 0.04 & 0.01 \\
\hline \hline
\end{tabular}

of 7. The Brillouin zone sampling ( $k$ mesh) was tested for convergence for each considered unit cell. In the more time-consuming computations (BSE, $G W$ ), we used $k$-mesh sizes of $6 \times 6 \times 6$ and $3 \times 3 \times 3$ for $\mathrm{Li}_{16} \mathrm{~F}_{15}$ and $\mathrm{Li}_{32} \mathrm{~F}_{31}$, respectively. The calculations for the $F$-center structures were carried out spin-polarized. For all our calculations we use the experimental lattice constant [11] of $4.03 \AA$ A. Defect-formation energies and structure relaxations were calculated using the PBE exchange-correlation functional [41]. Upon removal of a single fluorine atom in the unit cell, mainly the next-neighbor lithium and fluorine atoms tend to move away from the vacant fluorine site (Table I). These relaxations are small compared to the removal energy, $E_{\text {rem }}$, and the corresponding relaxation energy, $E_{\text {rel }}$, does not significantly affect the vacancy formation energy, $E_{\text {form }}=E_{\text {rem }}+E_{\text {rel }}$. The formation energies and local relaxations around the vacancy converge quickly with respect to the supercell size, and the band structures show only weak interactions between vacancies from neighboring unit cells (the flat vacancy band in Fig. 3 has a width of $0.12 \mathrm{eV}$ due to spurious vacancy-vacancy interactions in the 31-atom cell). We thus use the fully relaxed $\mathrm{Li}_{16} \mathrm{~F}_{15}$ structure as the starting point for the more time- and memory-consuming many-body calculations.

\section{B. Electronic band structure}

Since the quality of the $F$-center description also depends sensitively on the quality of the underlying bulk LiF electronic structure, we carefully checked the performance of different approximations on the quasiparticle band structure of $\mathrm{LiF}$. As for the electronic structure of pristine $\mathrm{LiF}$, the PBE eigenenergies show a band gap of $9.07 \mathrm{eV}$, underestimating by far the experimental gap of $14.2 \mathrm{eV}$ (Table II). Similarly, the Hartree-Fock approximation severely overestimates the gap by approximately $7 \mathrm{eV}$. In addition, we performed self-consistent hybrid-functional calculations involving the YS-PBE0 functional with $\alpha=0.25$ controlling the fraction of exact exchange. These calculations still underestimate the band gap by approximately $3 \mathrm{eV}$. For a more reliable prediction of the quasiparticle energies, we compare two approaches:

(i) the TB-mBJ potential [33] within the parametrization from Ref. [42]. The original Becke-Johnson (BJ) potential [43] is an approximation to the exact exchange potential obtained by the optimized-effective-potential (OEP) method for free
TABLE II. Calculated band gaps (in eV) for LiF. The PBE, $G W_{0} @ \mathrm{PBE}$, and $G_{0} W_{0} @ \mathrm{PBE}$ gaps are in good agreement with plane-wave results in Ref. [47] (given in parentheses) for a slightly different lattice parameter of $4.01 \AA$. The YS-PBE0 gap agrees well with the HSE06 value (given in parentheses) from Ref. [48]. Experimental gap and error estimate from Ref. [11].

\begin{tabular}{lcc}
\hline \hline PBE & 9.07 & $(9.20)$ \\
YS-PBE0 & 11.35 & $(11.47)$ \\
$G_{0} W_{0} @$ PBE & 12.96 & $(13.27)$ \\
$G W_{0} @$ PBE & 13.62 & $(13.96)$ \\
TB-mBJ & 14.51 & \\
Hartree-Fock & 21.16 & \\
Experiment & $14.2(2)$ & \\
\hline \hline
\end{tabular}

atoms [44], but it gives only small improvements for the band gap in solids $[33,42]$. TB-mBJ is an improvement to the BJ potential involving three empirical parameters [45] adjusted so that it gives the best band gap for a large number of compounds. With this choice of functional, we obtain a fairly accurate gap of $14.51 \mathrm{eV}$.

(ii) $G W$ calculations [23] have been performed using the GAP-FHI code [46]. $G W$ is an approximation to the self-energy obtained perturbatively from the Kohn-Sham orbitals. We applied it on top of PBE orbitals in the non-self-consistent form $\left(G_{0} W_{0} @ \mathrm{PBE}\right)$ and in the partially self-consistent form ( $G W_{0} @$ PBE, updating the energies in the Green's function). In the latter approximation, we obtain a quasiparticle gap of $13.62 \mathrm{eV}$. Since $G W_{0} @$ PBE gives slightly better agreement with experiment than $G_{0} W_{0} @ \mathrm{PBE}$, we will omit the discussion of $G_{0} W_{0} @ \mathrm{PBE}$ in the remainder of the text.

Turning now to the $F$ center in $\mathrm{LiF}$, the (spin-polarized) band structure of a $\mathrm{Li}_{16} \mathrm{~F}_{15}$ supercell is shown in Fig. 3 for four different calculation schemes, compared to the band structure of pristine $\mathrm{LiF}$ (calculated in the same supercell). The removal of a fluorine atom leaves the remaining electron of a lithium atom localized at the vacant site, which can be clearly seen from the wave function of the occupied $F$-center band shown in Fig. 2. Evidently it has almost pure $s$-character near the vacancy site. The corresponding band is located between 4.2 and $7.0 \mathrm{eV}$ above the valence-band edge (depending on the calculation scheme) and is almost flat. This is a good

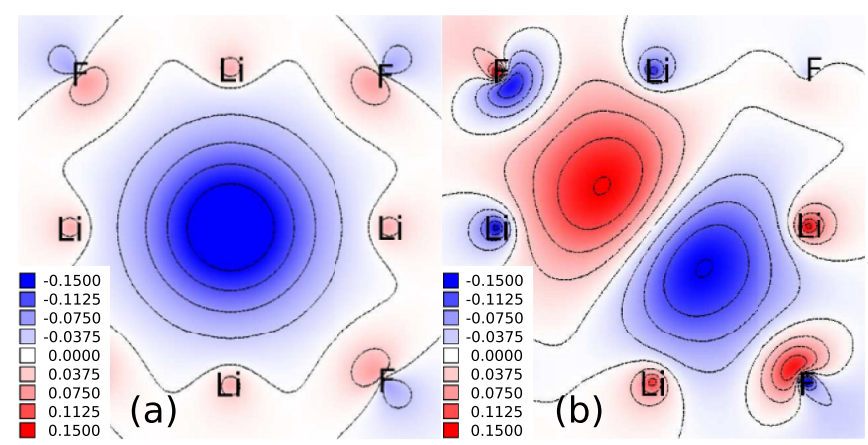

FIG. 2. (Color online) Kohn-Sham wave function (TB-mBJ) contour plot within the (100) plane at the $\Gamma$ point for the $(\alpha$-spin) $F$-center bands in the $\mathrm{Li}_{32} \mathrm{~F}_{31}$ supercell: (a) occupied $s$-orbital, (b) one of the three degenerate unoccupied $p$-orbitals. 

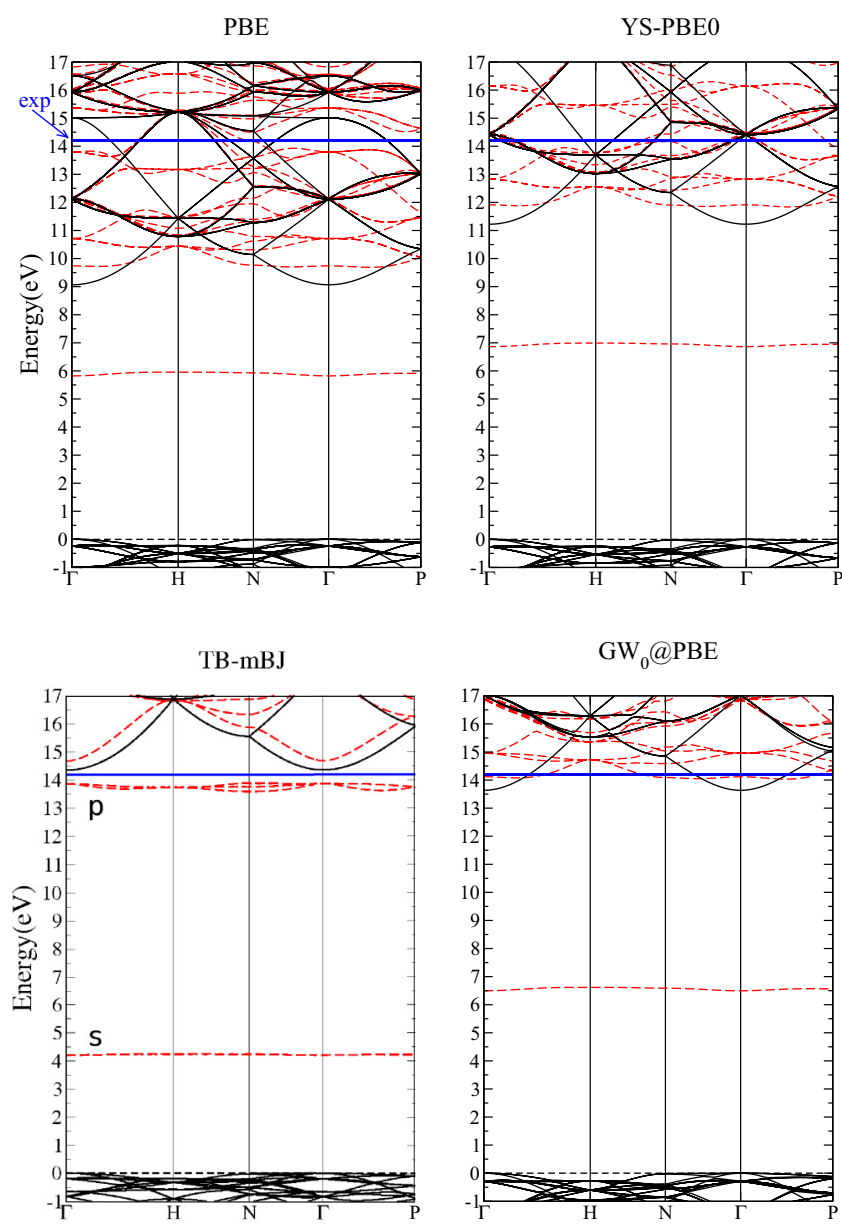

FIG. 3. (Color online) Band structures obtained from PBE, YS-PBE0, TB-mBJ, and $G W_{0} @$ PBE calculations. The black lines (full) show the band structure for perfectly crystalline lithium fluoride with $\mathrm{Li}_{16} \mathrm{~F}_{16}$ chosen as the unit cell. The red lines (dashed) show the highest occupied valence band and the conduction bands for the $F$-center structure (only $\alpha$-spin) from calculations where $\mathrm{Li}_{16} \mathrm{~F}_{15}$ was chosen as the unit cell. The blue line shows the experimental band gap of $14.2 \mathrm{eV}$.

indication that the supercell is large enough to describe the defect as strongly localized and well isolated. We also verified that the position of the impurity bands remain unchanged for larger supercells containing 128 and 256 atoms, respectively. A more critical test for the supercell size is the dispersion of the more delocalized conduction bands of the vacancy. They can be seen most clearly in the TB-mBJ band structure in Fig. 3 (labeled with " $p$ "). Their dispersion reduces from 0.3 to $0.1 \mathrm{eV}$ when we move from a 31-atom supercell to a 63-atom cell; the corresponding absorption spectra, however, are almost identical (the positions of the main peak differ by less than $0.1 \mathrm{eV}$ ). For $G W_{0} @ \mathrm{PBE}$ calculations, slightly larger deviations with respect to the cell size might be possible since the self-energy depends strongly on the underlying PBE bands, which are more diffuse than the TB-mBJ bands. Due to the very high computational cost of $G W_{0} @ \mathrm{PBE}$ and BSE calculations, we had to restrict the cell size to 31 atoms.

For PBE and YS-PBE0, the corresponding (unoccupied) $\beta$-spin band, which is not shown, is located $1.5 \mathrm{eV}$ above the $\alpha$-spin band within the band gap of LiF. In the case of $G W_{0} @ \mathrm{PBE}$, this separation is already of about $4.5 \mathrm{eV}$ (and the $\beta$-spin valence band still remains inside the gap), and for $\mathrm{TB}-\mathrm{mBJ}$ this band is dissolved within the conduction bands. Since selection rules in uv-vis absorption spectroscopy forbid spin flipping, we will not discuss $\beta$-spin band structures any further. The first excited defect state is threefold-degenerate at $\Gamma$ and resembles a $p$-orbital [see Fig. 2(b)]. On the DFT-PBE level, the band gap is too small (of about $5 \mathrm{eV}$ ) to accommodate this state as a discrete state, and therefore the defect $p$-band appears as a dispersive band in resonance with the conduction band. Using the YS-PBE0 potential does not change the dispersion of the conduction bands, and although it gives a $2.3 \mathrm{eV}$ better band gap for pristine $\mathrm{LiF}$, it only improves the $F$-center gap by $1.2 \mathrm{eV}$ since the occupied impurity band is shifted up by $1.1 \mathrm{eV}$ compared to PBE. On the $G W$ level, the band gap is strongly widened but the position of the defect $p$-band is shifted as well and it still appears as a resonant band within the conduction band. A similar observation was previously also made [27] for the $p$-band in $\mathrm{CaF}_{2}$. Interestingly, in the $\mathrm{TB}-\mathrm{mBJ}$ scheme, the defect $p$-band is located below the conduction-band edge and, consequently, it is only weakly dispersive. Only a self-consistent $G W$ calculation (including an update of the wave functions) can give the accurate position of the $p$-band relative to the conduction-band edge. For the calculation of optical spectra, however, inclusion of the strong electron-hole interaction upon excitation from the $s$ to the $p$ state turns out to be more important than the exact position of the $p$-band (see below).

\section{Absorption spectra}

In the independent-particle approximation (IPA), the position of the $F$-center absorption peak would be given by the energy difference between the $s$ - and the $p$-band. The resulting absorption spectra (imaginary parts of the dielectric function $\epsilon_{2}$ ) are shown in the upper part of Fig. 4. These results suggest that the absorption maxima of PBE and YS-PBE0 are at 4.5 and $5.5 \mathrm{eV}$, respectively, close to the experimental absorption maximum of $\sim 5 \mathrm{eV}$, while TB-mBJ and $G W_{0} @$ PBE overestimate it by several eV. We emphasize that using the PBE or YS-PBE0 exchange-correlation potential in the IPA seems to reproduce the absorption maxima in many cases quite well; however, it describes the wrong physics. The agreement with experiment must be considered accidental, as was recently also shown for the $F$ center [49] in $\mathrm{MgF}_{2}$. The IPA neglects the strong Coulomb attraction between the hole in the $s$-state and the electron in the $p$-state. These excitonic effects, which are taken into account by solving the Bethe-Salpeter equation (BSE) [25,26,37,38], significantly change the structure and position of the absorption peaks. This effect depends on the degree of localization of the valence and conduction states and is expected to be large in cases in which those states are well localized, for instance layered compounds [50] or excitation from core levels [51]. The absorption spectrum including the BSE is shown in the lower part of Fig. 4 for various underlying single-particle approximations. Compared to the measured spectrum, the PBE and YS-PBE0 spectra clearly underestimate the position of the absorption 


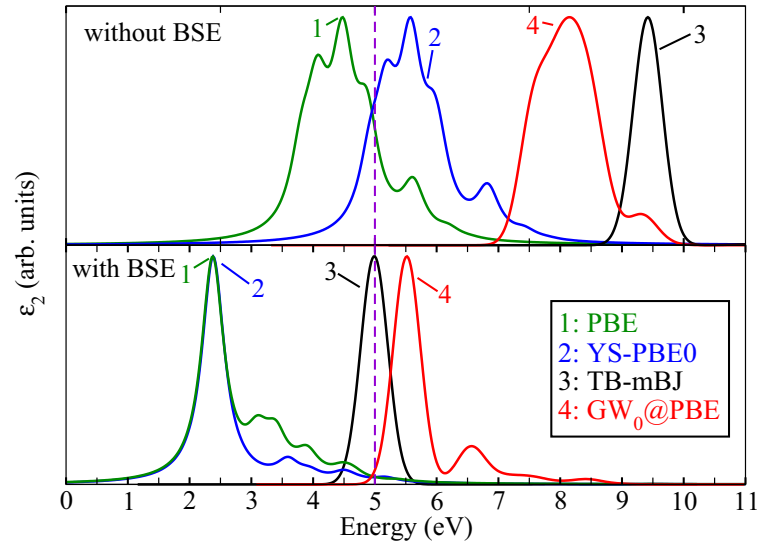

FIG. 4. (Color online) Imaginary part of the dielectric function $\left(\epsilon_{2}\right)$ scaled to equal peak height. Top: In the independent-particle approximation (neglecting electron-hole interactions). Bottom: Results with electron-hole interactions taken into account by solving the Bethe-Salpeter equation. $\mathrm{Li}_{16} \mathrm{~F}_{15}$ was chosen as the unit cell. The dashed line indicates the peak position of experimental absorption spectra.

peak by about $2.5 \mathrm{eV}$. This is due to the strong underestimation of the band gap and thus also of the $s-p$ transition energy. The $G W_{0} @ \mathrm{PBE}$ spectrum is blueshifted with respect to the PBE spectrum by about $3 \mathrm{eV}$ and thus matches the experimental spectrum quite well. In the independent-particle approximation, the main absorption peak would be at about $8.0 \mathrm{eV}$. The downshift to about $5.5 \mathrm{eV}$ is thus due to the very strong electron-hole attraction between the $s$ and $p$ states, which are both localized and thus very close to each other. The TB-mBJ calculations yield an independent-particle transition energy even $1.5 \mathrm{eV}$ higher than for $G W_{0} @ \mathrm{PBE}$. At the same time, screening is weaker and thus the electron-hole attraction is stronger such that the resulting absorption peak is close to the experiment and to the one of $G W_{0} @ \mathrm{PBE}$. The spectrum obtained from TB-mBJ shows a single peak due to transitions between the discrete $s$ and $p$ states as also present in experiment. All other calculations show a large peak together with several weak absorption features appearing as tails at higher energies. This additional side structure is related to the hybridization of the $p$-level with the conduction band. We expect this fine structure to change (or disappear entirely) upon a fully self-consistent $G W$ calculation that would also change and likely reduce the hybridization of the defect states with the conduction-band states. The absorption maximum from TB-mBJ and the absorption maximum of the first peak from $G W_{0} @$ PBE both lie within $0.5 \mathrm{eV}$ of the experiment.

\section{THE QUANTUM-CHEMISTRY APPROACH}

The present results from the periodic supercell approach as well as experimental results [34] suggest that the color center is a localized defect and its properties are only influenced by a limited number of atoms or ions surrounding it. Such a high degree of localization suggests that the $F$ center should be well described by quantum-chemistry methods within the framework of the embedded-cluster approach.

\section{A. Previous work}

The embedded-cluster approach has its origin in early single-electron model studies in which the vacancy electron is treated as a hydrogenic system embedded in a matrix of point charges [52]. One of the first ab initio SCF [restricted open-shell Hartree-Fock (ROHF)] calculations for the $F$ center in $\mathrm{LiF}$ was performed by Murrell and Tennyson [53]. They used a $\mathrm{Li}_{14} \mathrm{~F}_{12}$ cluster embedded in a Madelung potential represented by point charges. More advanced studies followed [17,18,54-56]. Kung, Kunz, and Vail [54] performed unrestricted Hartree-Fock (UHF) calculations for the six $\mathrm{Li}^{+}$ ions surrounding the vacancy embedded in a point-charge lattice. Pandey, Seel, and Kunz [55] extended this work by combining many-body perturbation theory with UHF calculations for various $\mathrm{LiF}$ clusters (up to $\mathrm{Li}_{14} \mathrm{~F}_{12}$ ) while neglecting embedding. Ewig and co-workers [17,18] presented a detailed ROHF study of a $\mathrm{Li}_{14} \mathrm{~F}_{12}$ cluster embedded in a point-charge lattice including results for the shape of the orbital wave function of the vacancy electron. For the same cluster size and similar embedding, Bader and Platts [56] investigated the topology of the ground-state electron density in the $F$ center. Furthermore, several DFT studies of the $F$ center in $\mathrm{LiF}$ based on the embedded-cluster approach [15,56] have been performed. A common feature of all these studies is that the $F$-center absorption energy is still in unsatisfying agreement with experimental results, which is surprising in view of the fact that the $F$ center is so well "atomiclike" localized. We therefore perform a detailed study of the $F$ center in $\mathrm{LiF}$ exploring possible sources of errors, including limited cluster size and basis sets, inadequate embedding, neglect of correlation, and electron-phonon coupling. While for the $F$ centers in other materials more accurate calculations using correlated methods are available (see, e.g., [57-59]), they are missing for the $F$ center in $\mathrm{LiF}$.

\section{B. Embedding scheme and basis sets}

In our study, we used active clusters of the sizes (1) $\mathrm{Li}_{14} \mathrm{~F}_{12}$, (2) $\mathrm{Li}_{38} \mathrm{~F}_{18}$, (3) $\mathrm{Li}_{62} \mathrm{~F}_{62}$, and (4) $\mathrm{Li}_{92} \mathrm{~F}_{86}$. We refer to these clusters as the small (1), medium (2), large (3), and very large cluster (4), respectively. The small cluster 1 is a cube with a side length of three ions with the vacancy located at the center. Adding one fluoride and four lithium ions on every side of the cube leads to the medium cluster 2 . The large cluster 3 is again a cube with a side length of five ions, and the very large cluster 4 is obtained by adding another four fluoride ions and five lithium ions on every side of the large cluster. For proper embedding, we use several layers of $a b$ initio model potentials (AIMPs) $[60,61]$ and a large matrix of point charges of cubic shape arranged as proposed by Evjen [62] with fractional charges of $+/-0.5,0.25$, and 0.125 at faces, edges, and corners, respectively. AIMPs are all-electron potentials in which, in contrast to pure point charges, also exchange terms are included by using nonlocal potentials. Exchange leads to repulsive forces between electrons of equal spin, or in this case, between the active electrons and the "frozen" electrons of the AIMPs. Their use is crucial in order to prevent unphysical excessive polarization of the active anions due to neighboring point charges and leakage of the electron cloud out of the region of the active cluster. For every cluster 
the size of AIMP and point charge embedding was chosen such that convergence of the absorption energy is reached. For the large cluster, which has cubic shape, we used four layers of AIMPs (2072 in total) and five layers of point charges (9970 in total) enclosing the active region. We used Dunning's correlation-consistent polarized valence-only basis sets [63] of double, triple, and quadruple zeta quality (cc-pVDZ, cc-pVTZ, and cc-pVQZ), which are referred to in the following as the small, medium, and large basis set. In addition to the basis sets localized at the ionic sites, we also placed a set of basis states pertaining to the $\mathrm{F}$ atom at the vacancy site. We note, however, that in line with earlier studies [56,57,64], this latter basis set only has a small effect on the convergence. Orbital size, shape, and energy as well as the absorption energy hardly change (the latter by less than $0.01 \mathrm{eV}$ ) compared to calculations with no additional vacancy basis set. This suggests that the defect wave function is well represented by basis states localized on ions surrounding the vacancy.

\section{Quantum-chemistry methods and cluster size}

On the Hartree-Fock level, the absorption energy of the cluster is calculated as the energy difference of two restricted open-shell Hartree-Fock (ROHF) $N$-electron wave functions with different symmetries corresponding to the ground state and the first optically allowed excited state. Similar to the findings of Adachi [64] for the $F$ center in $\mathrm{NaCl}$, we did not find a significant difference in the orbital shape and absorption energy between ROHF and unrestricted HF (UHF) calculations. Pristine LiF is a closed-shell system and, therefore, the unpaired electron attributed to the vacancy solely determines the symmetry of the many-body wave function of the cluster. Within the $D_{2 h}$ point-group description, the occupied ground-state orbital has $A_{g}$ symmetry while the excited state has either $B_{1 u}, B_{2 u}$, or $B_{3 u}$ symmetry. The ROHF $A_{g}$ and one of the $B_{u}$ orbitals are plotted in Figs. 5(a) and 5(b), respectively. Qualitatively, they resemble a hydrogen $1 \mathrm{~s}$ and $2 p$ wave function. For Fig. 5, the medium cluster size and the medium basis-set size were used. We note, however, that the size and shape of the $A_{g}$ and $B_{u}$ orbitals are well converged as a function of cluster and basis-set size.

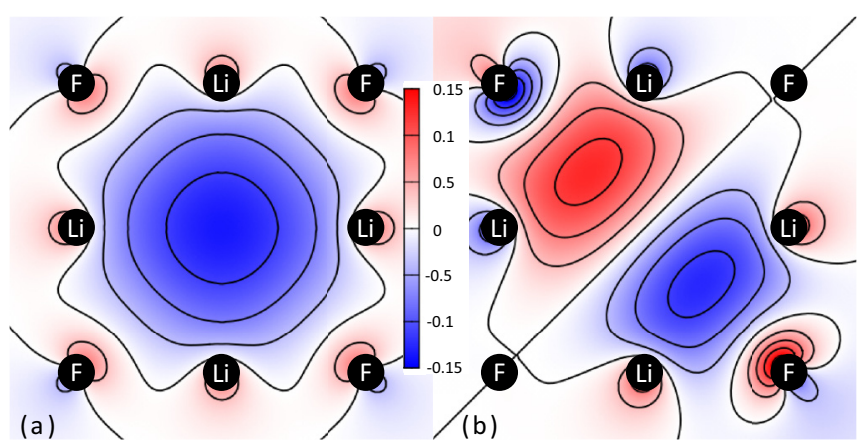

FIG. 5. (Color online) The ROHF orbitals of the electron in the $F$-center vacancy for the (a) ground state ( $s$-state) and (b) excited state ( $p$-state) configuration. Orbitals are taken from a calculation using the (medium) $\mathrm{Li}_{38} \mathrm{~F}_{18}$ cluster and the (medium) cc-pVTZ basis set.
While we find agreement with a previous calculation [18] for the $s$-state, the shape of the $p$-state is remarkably different. In Ref. [18], the $p$-state is strongly delocalized and spread over adjacent lattice sites, which may be the result of the pure point charge embedding. HF orbitals resulting from AIMP embedding are much more compact and localized in the vacancy region [65]. Similarly, the influence of the AIMPs on the absorption energy can be understood by means of the particle-in-the-box model. While pure point-charge embedding usually underestimates the absorption energy due to the diffuse " $p$ "-type orbitals, the inclusion of additional repulsive forces (exchange) in the embedding narrows the width of the box and leads to a larger separation of energy levels, i.e., to an increase of the absorption energy.

Quantum chemistry offers a large toolbox of methods beyond the Hartree-Fock level allowing for the inclusion of correlations. We apply methods specifically suited for accounting for dynamical correlation: complete active space second-order perturbation theory based on a single ROHF determinant CASPT2(ROHF) [a generalization of secondorder Møller-Plesset perturbation theory (MP2)], the coupled cluster single-double (CCSD), and the coupled cluster singledouble-perturbative triple [CCSD(T)] methods. For this group, the starting point is the ROHF wave function of either the ground or the excited state. We have also checked on the influence of static correlation by applying the complete active space self-consistent field (CASSCF) method and secondorder perturbation theory based on a multideterminant wave function [CASPT2(CAS)]. The CAS size for the ground state ( $A_{g}$ symmetry) was determined by correlating all occupied valence orbitals (F-2 $p$ orbitals) of $A_{g}$ symmetry plus a number of virtual orbitals also of $A_{g}$ symmetry. For the excited state, the same procedure was applied within one of the $B_{u}$ symmetries. For the medium cluster and medium basis set, the largest CASs tested were $(19,13)$ corresponding to 19 electrons in 13 orbitals and $(17,11)$ for the ground and excited state, respectively, leading to only small shifts of the total energies. This indicates the strong dominance of a single configuration. The effect of static correlation, i.e., the difference between CASPT2(CAS) and CASPT2(ROHF), on the absorption energy is a decrease of less than $0.02 \mathrm{eV}$.

To find the "suitable" method to treat dynamic correlation for the $F$ center in LiF, we benchmarked the CASPT2(ROHF), CCSD, and CCSD(T) as implemented in the MOLCAS 7.8 program package [66] striving for a compromise between computational cost and accuracy. HF, CASPT2(ROHF), CCSD, and $\mathrm{CCSD}(\mathrm{T})$ excitation energies of the small cluster are given in Table III for different basis sets. Note that CCSD and CCSD(T)

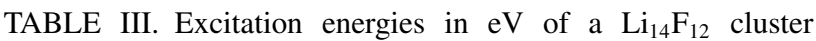
calculated with different methods and basis sets using the unperturbed crystal geometry and the experimental lattice constant.

\begin{tabular}{lcccc}
\hline \hline Basis set & ROHF & CASPT2(ROHF) & CCSD & CCSD(T) \\
\hline cc-pVDZ & 6.31 & 5.99 & 6.00 & 5.94 \\
cc-pVTZ & 6.27 & 5.84 & 5.86 & \\
cc-pVQZ & 6.26 & 5.79 & & \\
CBS limit & 6.26 & 5.73 & 5.77 & \\
\hline \hline
\end{tabular}


TABLE IV. CASPT2(ROHF) excitation energies in eV calculated with different cluster sizes and basis sets using the unperturbed crystal geometry and the experimental lattice constant.

\begin{tabular}{lcccc}
\hline \hline Basis set & $\mathrm{Li}_{14} \mathrm{~F}_{12}$ & $\mathrm{Li}_{38} \mathrm{~F}_{18}$ & $\mathrm{Li}_{62} \mathrm{~F}_{62}$ & $\mathrm{Li}_{92} \mathrm{~F}_{86}$ \\
\hline cc-pVDZ & 5.99 & 5.87 & 5.76 & 5.74 \\
cc-pVTZ & 5.84 & 5.73 & 5.61 & \\
cc-pVQZ & 5.79 & 5.70 & & \\
CBS limit & 5.73 & 5.63 & 5.50 & \\
\hline \hline
\end{tabular}

could not be calculated for the larger basis sets. The last line in Table III shows values for the converged basis set (CBS) limit obtained by employing the extrapolation scheme proposed by Truhlar $[67,68]$. This scheme is tailored to extrapolate perturbation theory, CCSD, and $\operatorname{CCSD}(\mathrm{T})$ energies from the cc-pVDZ and cc-pVTZ basis sets to the CBS limit allowing for application to cases in which cc-pVQZ calculations were not possible. Where available, we compare the Truhlar CBS energies to values from extrapolation schemes involving the energies of the larger cc-pVQZ basis set [69], which show a deviation of less than $0.01 \mathrm{eV}$ in the excitation energy. Table III shows that the CASPT2(ROHF) method closely reproduces the excitation energy determined by methods that include correlations to a larger degree, such as CCSD and CCSD(T). For example, the CBS limits of the CASPT2(ROHF) and the CCSD differ by only $0.04 \mathrm{eV}$. A comparison between CASPT2(ROHF) and CCSD(T) excitation energies is only possible for the small basis set. In this case, the $\operatorname{CCSD}(\mathrm{T})$ excitation energy is $0.05 \mathrm{eV}$ lower than the CASPT2(ROHF) value. In view of these negligible deviations $(\lesssim 1 \%$ of the experimental excitation energies), we employ the numerically relatively cheap CASPT2(ROHF) method to larger clusters to check for cluster-size convergence. The CASPT2(ROHF) excitation energies for the different cluster sizes and the different basis sets as well as the extrapolated CBS values are given in Table IV. Since for the small basis set the difference between the large and very large cluster is only $0.02 \mathrm{eV}$, the excitation energy calculated for the large cluster is considered to be converged within a satisfactory level of accuracy. All results given in the following refer to the CBS limit of the large cluster unless otherwise stated.

One possibility to study the effect of correlations on single-particle orbitals is the construction of natural orbitals that diagonalize the exact first-order density matrix. The difference of the $s$ - and $p$-type first-order natural orbitals to the corresponding HF orbitals (Fig. 5) is approximately three orders of magnitude smaller than their actual value. However, the difference between their absolute magnitudes squared in Figs. 6(a) and 6(b) for $A_{g}$ and $B_{u}$ symmetry, respectively, indicates that correlation tends to slightly delocalize the $F$ center $A_{g}$ and $B_{u}$ orbitals and shifts electron density from the vacancy site to the surrounding $\mathrm{F}^{-}$ions.

\section{Relaxation of the ground state-The Franck-Condon absorption energy}

The convergence tests discussed above were performed for an unperturbed crystal structure using the experimental value of the lattice constant. For comparison with experimental

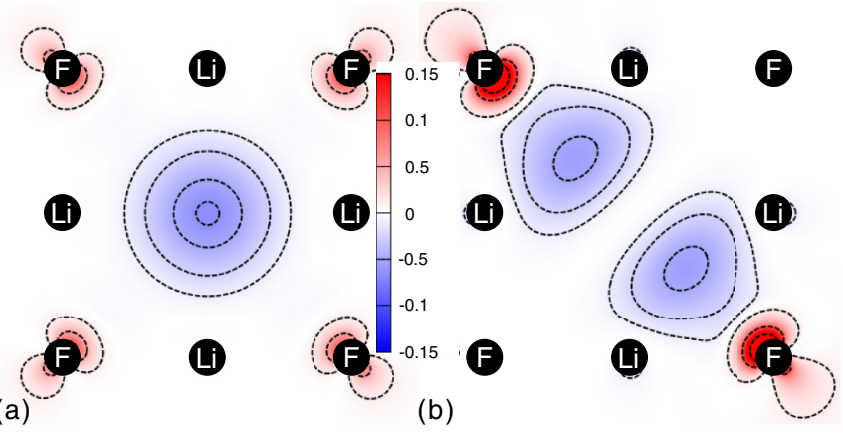

FIG. 6. (Color online) Density difference $\Delta=\left|\phi_{\text {nat }}\right|^{2}-\left|\phi_{\mathrm{HF}}\right|^{2}$ in $10^{-4} /$ bohr $^{3}$ between the absolute magnitudes of the first-order natural orbitals and the Hartree-Fock orbitals of the electron in the $F$-center vacancy for the (a) ground state ( $s$-state) and (b) excited state ( $p$-state) configuration. Orbitals from calculations using the (medium) $\mathrm{Li}_{38} \mathrm{~F}_{18}$ cluster and the (medium) cc-pVTZ basis set are shown.

absorption spectra, we have studied the $F$ center including geometry relaxation. One advantage of wave-function-based methods is that state-specific relaxation can be performed, i.e., different relaxed geometries for the ground and the excited states can be determined. In the relaxed geometry for the ground state, the ground- and excited-state energy were calculated to simulate a vertical, Franck-Condon-like, excitation. The geometry relaxation was performed on the CASPT2(ROHF) level using the medium cluster size and the medium basis set under the constraint that only nearest and next nearest neighbors were allowed to move. This constraint was imposed for two reasons. First, the more general (periodic boundary) DFT relaxation showed essentially no movement of ions farther away. Second, potentially unphysical relaxation of ions in the outermost layer of the active cluster neighboring AIMPs should be avoided. Similar to the DFT relaxation, we found $0.044 \AA$ outward movement of the $\mathrm{Li}^{+}$ions and $0.024 \AA$ outward movement of the $\mathrm{F}^{-}$ions. Using the relaxed geometry, we find in the CBS limit an HF excitation energy of $5.96 \mathrm{eV}$ and a CASPT2(ROHF) excitation energy of $5.31 \mathrm{eV}$. This corresponds to a decrease of the absorption energy due to the relaxation of $0.21 \mathrm{eV}$ for the $\mathrm{HF}$ case and $0.19 \mathrm{eV}$ for the CASPT2(ROHF) case, respectively.

\section{E. Linewidth}

The linewidth of the $F$-center absorption in alkali halides is significantly influenced by electron-phonon interactions. Lifetime broadening can be neglected due to the long lifetime [34] of up to $\sim 10^{-6} \mathrm{~s}$ of the excited state. Typically one local mode defining the relevant configuration coordinate dominates the linewidth and the absorption process $[7,70]$. In the present case, this mode is the symmetric breathing mode of the six $\mathrm{Li}^{+}$ions surrounding the vacancy (inset in Fig. 7). Using the medium basis set and medium cluster size, we have calculated the configuration coordinate diagram of this mode for the ground and excited state of the $F$ center (Fig. 7) and extracted a vibration frequency $(15.78 \mathrm{THz}, 65.2 \mathrm{meV})$ and a linewidth of $0.27 \mathrm{eV}$ due to the zero-point fluctuations. Stoneham [7] proposed, as a "rule of thumb", that the frequency of this mode is comparable to the transverse optical phonon 


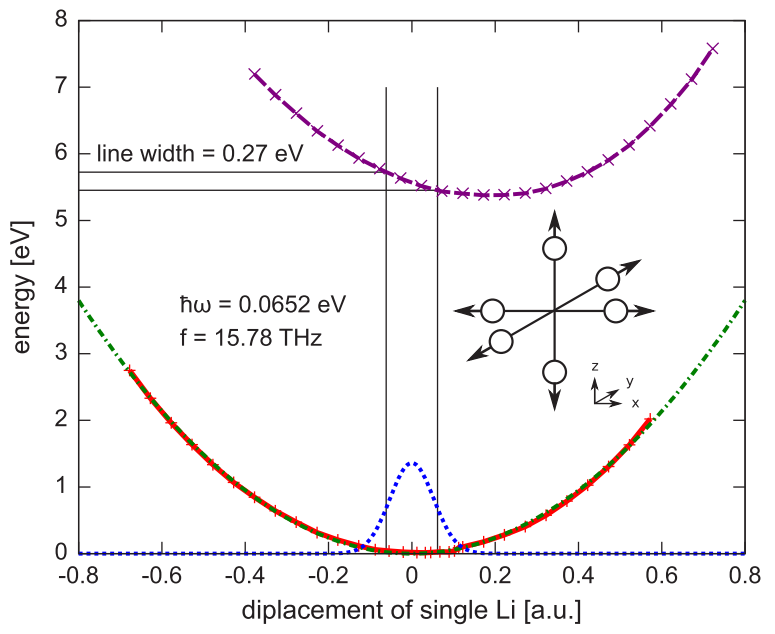

FIG. 7. (Color online) Calculated configuration coordinate curves for the ground (red full line) and excited (violet dashed) state of the $F$ center in $\mathrm{LiF}$ as a function of the elongation along the symmetric breathing vibration of the six $\mathrm{Li}^{+}$ions surrounding the defect for the (medium) $\mathrm{Li}_{38} \mathrm{~F}_{18}$ cluster and the (medium) cc-pVTZ basis set. The blue (dotted) line is the absolute magnitude squared of the ground-state wave function of a harmonic potential with $\hbar \omega=0.0652 \mathrm{eV}$ (green dash-dotted line). The inset schematically depicts the symmetric breathing vibration of the six $\mathrm{Li}^{+}$ions surrounding the defect.

frequency of the bulk material. For pristine LiF, the TO modes lie at $\sim 10 \mathrm{THz}[71,72]$, which compares reasonably well with our estimates. Also the resulting theoretical linewidth extracted from the configuration coordinate curves is in fair agreement with the experimental values [12,73] at low temperatures ranging from 0.42 to $0.61 \mathrm{eV}$. This single-mode estimate for the linewidth should be considered as a lower bound to the experimental linewidth. Possible effects that would further increase the theoretical linewidth are additional modes comprising ions beyond the nearest neighbors, thermal broadening, and broadening due to imperfections of the crystal (inhomogeneous broadening). Additionally, folding the line with experimental resolution would further increase the linewidth.

\section{F. Relaxation of the excited state}

Exploiting the tool of state-specific relaxation available within a quantum-chemistry approach, we have studied the properties of the relaxed $2 p$ state. For the unperturbed crystal geometry (and also for the relaxed ground-state geometry) there are, within the $D_{2 h}$ point-group symmetry, two energetically degenerate orientations of the $p$ orbital pointing either to the nearest neighbor $\mathrm{Li}^{+}$or to the nearest neighbor $\mathrm{F}^{-}$. Relaxing the geometry for both orientations, however, leads to a Jahn-Teller-like distortion lifting this degeneracy. The configuration in which the " $p$ "-type orbital points toward the $\mathrm{F}^{-}$ions is $\sim 0.12 \mathrm{eV}$ lower in energy than the one in which the orbital points toward the $\mathrm{Li}^{+}$ions. For both cases, the displacement from the unperturbed crystal geometry is shown in Fig. 8. We find maximum displacements of $\sim 0.23 \AA$. Such large displacements [70] are consistent with large Stokes shifts

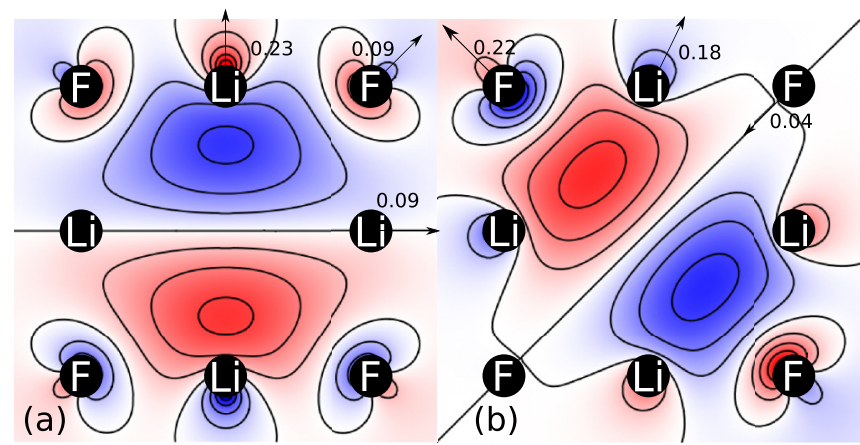

FIG. 8. (Color online) Cut through ROHF- $p$ molecular orbital oriented toward (a) nearest-neighbor $\mathrm{Li}^{+}$ions and (b) next-nearestneighbor $\mathrm{F}^{-}$ions. Arrows indicate the displacement of ions neighboring the $F$ center due to relaxation of the excited state. Their magnitude is given in $\AA$. Orbitals and displacements [on the CASPT2(ROHF) level] are calculated using the (medium) $\mathrm{Li}_{38} \mathrm{~F}_{18}$ cluster and the (medium) cc-pVTZ basis set.

of roughly $1 \mathrm{eV}$ between absorption and emission in alkali halides. Furthermore, similar values for the displacement for the transition from the $\mathrm{F}$ to the $\mathrm{F}^{2+}$ center were found [30] in $\mathrm{MgO}$. The relaxation of the excited state leads to a reduction of its total energy by $E_{\text {relax }} \approx 0.5 \mathrm{eV}$. However, we note that the values for the displacements and for the relaxation energy $E_{\text {relax }}$ may be affected by the limited cluster size since the outgoing $\mathrm{F}^{-}$ions are not moving toward active ions but rather toward AIMPs. An estimate based on experimental data [12] yields a relaxation energy of $\sim 1.6 \mathrm{eV}$. A definite answer on the excited-state geometry and its relaxation energy awaits further calculations with larger clusters and inclusion of long-range polarization effects.

\section{G. Electron-phonon coupling}

The coupling of electronic and nuclear degrees of freedom may lead to a redshift of the absorption line of the $F$ center with respect to its Franck-Condon value. We estimate contributions to this shift by analyzing the energy surfaces along configuration coordinates of the embedded cluster. Alternatively, they can be estimated from the energy shift of a "small" polaron.

From the variation of the electronic potential surface along an effective coordinate (Fig. 9), we extract as an upper bound for the shift $\Delta E_{e \text {-ph }}$ of the absorption energy the relaxation energy of the excited state $E_{\text {relax }}$, i.e., the difference between the Franck-Condon line ("vertical" excitation) and the zero phonon line ("nonvertical" excitation resulting in the lowest possible absorption energy). The zero-phonon line corresponds to the excitation from the minimum of the ground-state to the minimum of the excited-state energy (see below) within the multidimensional space used in our geometry relaxation. The difference between the zero-phonon line and the Franck-Condon line amounts to $E_{\text {relax }} \sim 0.5 \mathrm{eV}$. This is an upper bound $E_{\text {relax }}=\Delta E_{e-\mathrm{ph}}^{\max }$ to the contribution of electron-phonon coupling to the redshift of the absorption line. The real shift will be generally much lower. One estimate can be obtained by calculating the overlap of the nuclear wave 


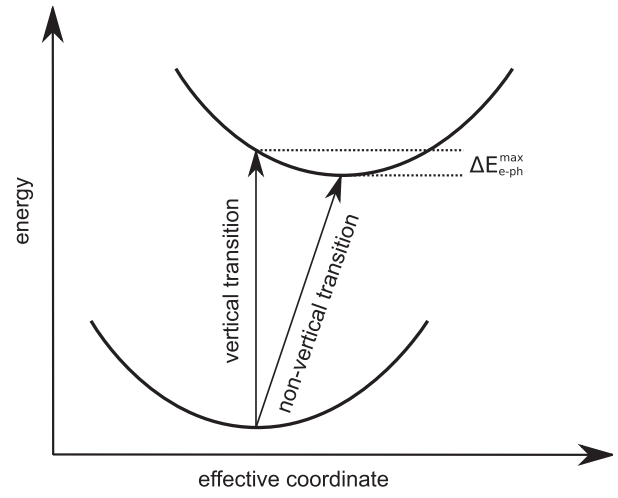

FIG. 9. Schematic picture of electronic potential energy surfaces of the ground and excited state of the $F$ center as a function of an effective coordinate. Arrows indicate the vertical (Franck-Condonlike) transition and the nonvertical transition to the minimum of the excited-state potential energy surface. The difference $\Delta E_{e-\mathrm{ph}}^{\max }$ in energy between these two excitations is an upper bound for the redshift of the absorption line due to electron-phonon interactions.

functions in the ground and excited states for the different $n$-phonon lines.

Alternatively, within a polaron model, the correction to an electronic energy level is given by the polaron self-energy induced by virtual excitations of electrons to the conduction band and their interaction with longitudinal optical phonons. Accordingly, differences between the polaron self-energies for the electronic ground and excited state of the color center contribute to the shift of the absorption line with respect to its Franck-Condon value. Since in alkali halides electron-phonon coupling is large, we employ Feynman's strong-coupling limit for the polaron self-energy [74] (in a.u. $), E_{\text {polaron }}=-\left(0.106 \alpha^{2}+2.83\right) \omega$, where $\alpha$ is the Fröhlich coupling constant [75] and $\omega$ is the longitudinal optical phonon frequency. We estimate the shift of the absorption line as $\Delta E_{e-\mathrm{ph}}^{\left(Q_{j}\right)}=-\left(0.106 \alpha^{2}+2.83\right) \Delta \omega_{Q_{j}}$ through the largest difference in vibration frequency $\Delta \omega_{Q_{j}}$ of the local normal mode $Q_{j}$ involving the six neighboring $\mathrm{Li}^{+}$ions between the ground and excited state of the color center. The frequencies were obtained from parabolic fits to configuration coordinate curves calculated with the medium cluster and the medium basis-set size. As a dominant contribution we find $\Delta \omega_{Q_{2}} \approx$ $0.015 \mathrm{eV}$, where $Q_{2}$ corresponds to the stretch vibration shown in Fig. 10. Using the Fröhlich coupling constant of pristine $\mathrm{LiF}$ $(\alpha=5.25)$ yields $\Delta E_{e-\mathrm{ph}}=0.09 \mathrm{eV}$, which is comparable to the difference of the relaxation energies of the excited state for the two $p$-type orbital orientations (Sec. IV D).

In addition, we have also explored dynamical corrections to the Born-Oppenheimer energy surfaces which scale with the inverse of the effective mass. The lowest-order non-BornOppenheimer (NBO) corrections $\Delta E^{(\mathrm{NBO})}$ are given by the eigenvalues of the Hamiltonian matrix

$$
\begin{aligned}
\Delta H_{i, j}= & -\sum_{a} \frac{1}{M_{a}} \int d R\left\langle\phi_{j}\left|\nabla_{a}\right| \phi_{i}\right\rangle \chi_{j} \nabla_{a} \chi_{i} \\
& +\int d R\left\langle\phi_{j}\left|T_{R}\right| \phi_{i}\right\rangle \chi_{j} \chi_{i},
\end{aligned}
$$

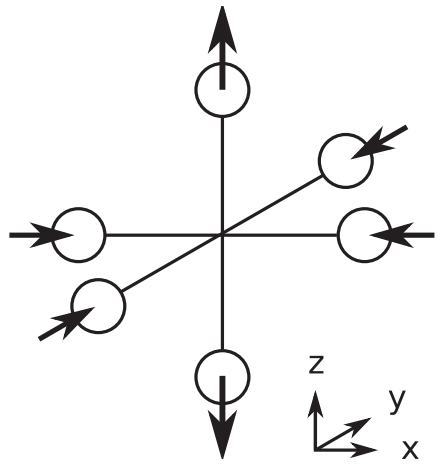

FIG. 10. Schematic picture of the stretch vibration of the six $\mathrm{Li}^{+}$ions neighboring the vacancy responsible for the dominant contribution $\Delta \omega_{Q_{2}} \approx 0.015 \mathrm{eV}$.

where $\chi_{i}$ is the nuclear wave function in the $i$ th electronic state,

$$
\begin{aligned}
T_{R} & =\sum_{a}-\frac{1}{2 M_{a}} \nabla_{a}^{2}, \\
\nabla_{a} & =\left(\frac{\partial}{\partial X_{a}}, \frac{\partial}{\partial Y_{a}}, \frac{\partial}{\partial Z_{a}}\right) .
\end{aligned}
$$

The index $a$ runs over the atoms in the embedded cluster. The shift of the $F$-center absorption line due to electron-vibrational coupling is then given by the difference $\Delta E^{(\mathrm{NBO})}=E_{p}^{(\mathrm{NBO})}-$ $E_{s}^{(\mathrm{NBO})}$. We estimate $\Delta E^{(\mathrm{NBO})}$ from a strongly truncated matrix including only the lowest-lying $s$ - and $p$-type states and with only the six-nearest-neighbor $\mathrm{Li}^{+}$ions closest to the vacancy allowed to vibrate. The electronic matrix elements were evaluated on the multistate CASPT2 level using finite differences, and the harmonic approximation was used for the nuclear wave functions $\chi_{i}$. As expected, this estimate leads to a negligible energy shift below $0.0001 \mathrm{eV}$. We conclude that these dynamical corrections are completely negligible compared to the corrections due to quasistatic lattice distortion and relaxation.

\section{COMPARISON OF THE TWO APPROACHES AND WITH EXPERIMENTAL DATA}

The solid-state physics approach and the quantumchemistry approach can be compared on several levels. On the single-particle level, we can compare the Hartree-Fock and the Kohn-Sham orbitals generated with the TB-mBJ exchange-correlation potential. Both approaches lead to a similar structure of the single-particle levels in a ground-state calculation: the occupied $s$-type and unoccupied $p$-type levels lie within the band gap, and the corresponding orbitals are localized within the vacancy region. Both orbital pairs agree in size and shape (compare Figs. 2 and 5). We note that Fig. 5(b) represents an occupied HF $p$-type orbital while Fig. 2(b) depicts an unoccupied Kohn-Sham orbital. However, the corresponding unoccupied ROHF- $p$ orbital in the presence of an occupied $s$ orbital looks qualitatively like the orbital in Fig. 5(b).

We also find good agreement between the two approaches concerning the ground-state relaxation of the nearest and 


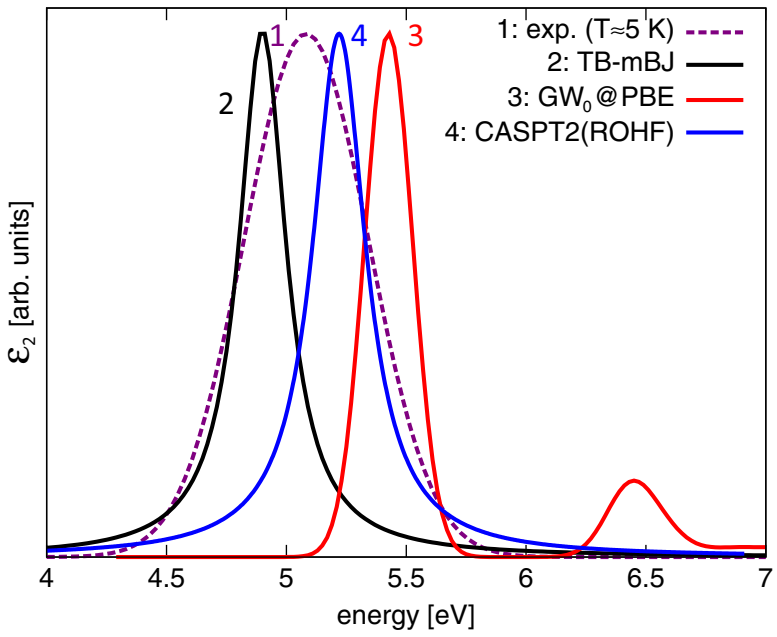

FIG. 11. (Color online) Experimental [12] (dashed line) and calculated (solid lines) absorption spectra of the $F$ center in LiF. The experimental spectrum is measured at $T \approx 5 \mathrm{~K}$ and is depicted as a Gaussian function with a peak position of $E_{p}=5.08 \mathrm{eV}$ and a full width at half-maximum of $0.61 \mathrm{eV}$. Spectra calculated from post-DFT methods (TB-mBJ + BSE and $G W_{0} @ \mathrm{PBE}+\mathrm{BSE}$ ) are determined within a $\mathrm{Li}_{16} \mathrm{~F}_{15}$ unit cell. The quantum-chemistry result is obtained from a CASPT2(ROHF) (CASPT2 with a single ROHF determinant) calculation in the converged basis-set limit of a $\mathrm{Li}_{62} \mathrm{~F}_{62}$ embedded cluster. All theoretical curves are plotted with a calculated linewidth at zero temperature (Sec. IV E) of $0.27 \mathrm{eV}$, and they contain a redshift due to electron-phonon coupling of $\Delta E_{e-\mathrm{ph}}=0.09 \mathrm{eV}$ (Sec. IV G).

next-nearest neighbors surrounding the $F$ center. DFT and CASPT2(ROHF) lead to an identical outward relaxation of $0.04 \AA$ of the nearest-neighbor $\mathrm{Li}^{+}$ions and to similarly small outward relaxation of the $\mathrm{F}^{-}$ions $[0.01 \AA$ in DFT and $0.024 \AA$ in CASPT2(ROHF)]. The small discrepancy for the $\mathrm{F}^{-}$ions might be due in part to the limited cluster size for which the CASPT2(ROHF) geometry relaxation was performed (see Sec. IV D). We note that such an agreement between periodic DFT and quantum-chemistry cluster calculations is not standard. For example, for the $F$ center in $\mathrm{MgO}$ the relaxation of the $\mathrm{Mg}^{+}$ion obtained from periodic DFT [30] and the relaxation from cluster calculations on the HF level [58] differ by a factor of $\sim 5$.

In Fig. 11, we present a comparison between the experimental absorption spectrum [12] at $T \approx 5 \mathrm{~K}$ and absorption spectra obtained by the quantum-chemistry and the solid-state physics approaches. The experimental spectrum is represented by a Gaussian distribution with parameters for peak position $E_{p}=$ $5.08 \mathrm{eV}$ and a full width at half-maximum (FWHM) of $0.61 \mathrm{eV}$. This peak position is a blueshift relative to the experimental spectrum at room temperature [12] by $\Delta E_{T} \approx 0.14 \mathrm{eV}$. All theoretical spectra include the calculated zero-temperature linewidth of $0.27 \mathrm{eV}$ (Sec. IVE) and are shifted toward lower energies by $\Delta E_{e \text {-ph }}=0.09 \mathrm{eV}$ (Sec. IV G) due to the influence of electron-phonon coupling. Overall, the calculated absorption spectra with peaks at $5.22 \mathrm{eV}$ [CASPT2(ROHF)], $5.42 \mathrm{eV}\left(G W_{0} @ \mathrm{PBE}+\mathrm{BSE}\right)$, and $4.9 \mathrm{eV}(\mathrm{TB}-\mathrm{mBJ}+\mathrm{BSE})$ show unprecedented agreement with the experimental data for the $F$-center absorption spectrum of $\mathrm{LiF}$.
The complementarity of the two approaches allows us to disentangle the origin of the corrections to the absorption energy, namely corrections due to excitonic and correlation effects. In the solid-state physics approach, correlation is included to a large degree on the DFT level either by the choice of a "good" exchange-correlation potential or by the application of the $G W$ method. The largest residual error is the neglect of excitonic effects, that is, the screened Coulomb interaction of the electron in the excited $p$-state with the hole in the $s$-state. This electron-hole interaction can be included by employing the Bethe-Salpeter equation. The correction due to the BS equation amounts to a lowering of the absorption energy by approximately $3 \mathrm{eV}$ for $G W_{0} @ \mathrm{PBE}$ and by $4 \mathrm{eV}$ for the DFT calculation using the TB-mBJ exchange-correlation potential but without $G W$. The attractive hole lowers the energy of the electron in the excited state.

In the quantum-chemistry approach, on the other hand, the electron-hole pair interaction is included already on the Hartree-Fock level since different configurations are explicitly used for the ground and excited state. The correlation energy, however, has to be accounted for in an additional step. As shown in Sec. IV C, the CASPT2(ROHF) method gives the correlation energy correction to a very good degree of approximation, amounting to about $0.65 \mathrm{eV}$ and also lowering the absorption energy.

\section{CONCLUSION}

We have revisited the $F$ color center in LiF with stateof-the-art ab initio methods employed in both the solid-state physics and quantum-chemistry context. Due to the strong localization of both ground $(s)$ and first excited $(p)$ states, both the periodic supercell approach and the embedded-cluster approach are suitable to treat the problem with high accuracy. In the periodic supercell approach, starting from DFT wave functions and energies, the methods of many-body perturbation theory ( $G W$ approximation and BSE) are required to overcome the underestimation of the band gap and the neglect of electron-hole interaction. The latter amounts to almost $4 \mathrm{eV}$ in the present case. We have shown that the TB-mBJ functional provides a very good starting point for the calculation of absorption spectra that allows us to bypass the $G W$ correction. Nevertheless, the solution of the Bethe-Salpeter equation is required to account for the strong excitonic effects in $\mathrm{LiF}$. The use of the Hartree-Fock approximation in the embedded-cluster approximation overestimates the absorption energy of the $F$ center. However, inclusion of dynamical correlation effects on the level of second-order perturbation theory yields results in close agreement with the $G W+$ Bethe-Salpeter calculations and with the position of the experimental absorption peaks. The present quantum-chemistry calculation provides furthermore a straightforward estimate of the electron-phonon-induced line broadening and shift.

Detailed comparisons of periodic supercell and embeddedcluster approaches are still rather scarce. Our present study demonstrates that they work equally well for strongly localized defects provided that correlation effects are properly taken into account. 


\section{ACKNOWLEDGMENTS}

We thank H. Jiang, G. Baldacchini, and F. Aquilante for discussions and technical support. This work was supported by the Austrian Fonds zur Förderung der wissenschaftlichen
Forschung (Projects SFB F41 "ViCoM" and the doctoral college W1243 "Solids4Fun"). F.K. and P.B. acknowledge the support by the TU Vienna doctoral college COMPMAT. L.W. acknowledges support by the National Research Fund, Luxembourg (Project C12/MS/3987081/TSDSN).
[1] G. Baldacchini and R. M. Montereali, Opt. Mat. 16, 53 (2001).

[2] V. V. Ter-Mikirtychev and T. Tsuboi, Prog. Quantum Electron. 20, 219 (1996).

[3] C. A. Hutchinson, Phys. Rev. 75, 1769 (1949).

[4] N. W. Lord, Phys. Rev. Lett. 1, 170 (1958).

[5] J. H. Schulman and W. D. Compton, Color Centers in Solids (Pergamon, New York, 1962).

[6] W. B. Fowler, The Physics of Colour Centers (Academic, New York, 1968).

[7] A. M. Stoneham, Theory of Defects in Solids (Clarendon, Oxford, 1975).

[8] L. V. E. Caldas, M. R. Mayhugh, and T. G. Stoebe, J. Appl. Phys. 54, 3431 (1983).

[9] G. Singh and T. E. Gallon, Solid State Commun. 51, 281 (1984).

[10] G. Roy, G. Singh, and T. E. Gallon, Surf. Sci. 152-153, 1042 (1985).

[11] M. Piacentini, D. W. Lynch, and C. G. Olson, Phys. Rev. B 13, 5530 (1976).

[12] R. K. Dawson and D. Pooley, Phys. Status Solidi B 35, 95 (1969).

[13] K. Schwartz, C. Trautmann, A. S. El-Said, R. Neumann, M. Toulemonde, and W. Knolle, Phys. Rev. B 70, 184104 (2004).

[14] G. Baldacchini, A. T. Davidson, V. S. Kalinov, A. G. Kozakiewicz, R. M. Montereali, E. Nichelatti, and A. P. Voitovich, J. Lumin. 122-123, 371 (2007).

[15] M. R. Pederson and B. M. Klein, Phys. Rev. B 37, 10319 (1988).

[16] G. Mallia, R. Orlando, C. Roetti, P. Ugliengo, and R. Dovesi, Phys. Rev. B 63, 235102 (2001).

[17] C. Kölmel and C. S. Ewig, J. Phys. Chem. B 105, 8538 (2001).

[18] C. S. Ewig, J. Tellinghuisen, and M. H. Mendenhall, Chem. Phys. Lett. 188, 501 (1992).

[19] A. S. Shalabi, A. M. El-Mahdy, M. A. Kamel, and H. Y. Ammar, Physica B 304, 444 (2001).

[20] C. S. Wang and B. M. Klein, Phys. Rev. B 24, 3393 (1981).

[21] C. S. Wang and B. M. Klein, Phys. Rev. B 24, 3417 (1981).

[22] J. Heyd, J. E. Peralta, G. E. Scuseria, and R. L. Martin, J. Chem. Phys. 123, 174101 (2005).

[23] L. Hedin, Phys. Rev. 139, A796 (1965).

[24] M.S. Hybertsen and S. G. Louie, Phys. Rev. B 34, 5390 (1986).

[25] M. Rohlfing and S. G. Louie, Phys. Rev. B 62, 4927 (2000).

[26] S. Albrecht, L. Reining, R. Del Sole, and G. Onida, Phys. Rev. Lett. 80, 4510 (1998).

[27] Y. Ma and M. Rohlfing, Phys. Rev. B 77, 115118 (2008).

[28] M. Bockstedte, A. Marini, O. Pankratov, and A. Rubio, Phys. Rev. Lett. 105, 026401 (2010)

[29] C. Attaccalite, M. Bockstedte, A. Marini, A. Rubio, and L. Wirtz, Phys. Rev. B 83, 144115 (2011).

[30] P. Rinke, A. Schleife, E. Kioupakis, A. Janotti, C. Rödl, F. Bechstedt, M. Scheffler, and C. G. Van de Walle, Phys. Rev. Lett. 108, 126404 (2012).
[31] J. Lischner, J. Deslippe, M. Jain, and S. G. Louie, Phys. Rev. Lett. 109, 036406 (2012).

[32] F. Tran and P. Blaha, Phys. Rev. B 83, 235118 (2011).

[33] F. Tran and P. Blaha, Phys. Rev. Lett. 102, 226401 (2009).

[34] G. Baldacchini, in Optical Properties of Excited States in Solids, NATO ASI Series Vol. 301, edited by B. Bartolo and C. Beckwith (Springer, New York, 1992), pp. 255-303.

[35] M. Fox, Optical Properties of Solids, 1st ed. (Oxford University Press, Oxford, 2001), pp. 186-203.

[36] W. G. Aulbur, L. Jönsson, and J. W. Wilkins, Solid State Phys. 54, 1 (1999).

[37] G. Onida, L. Reining, and A. Rubio, Rev. Mod. Phys. 74, 601 (2002).

[38] P. Puschnig, Ph.D. thesis, Institute of Theoretical Physics Universität Graz, 2002.

[39] J. J. Shepherd, A. Grüneis, G. H. Booth, G. Kresse, and A. Alavi, Phys. Rev. B 86, 035111 (2012).

[40] P. Blaha, K. Schwarz, G. K. H. Madsen, D. Kvasnicka, and J. Luitz, WIEN2K: An Augmented Plane Wave Plus Local Orbitals Program for Calculating Crystal Properties (Vienna University of Technology, Vienna, 2001).

[41] J. P. Perdew, K. Burke, and M. Ernzerhof, Phys. Rev. Lett. 77, 3865 (1996).

[42] D. Koller, F. Tran, and P. Blaha, Phys. Rev. B 83, 195134 (2011).

[43] A. D. Becke and E. R. Johnson, J. Chem. Phys. 124, 221101 (2006).

[44] J. D. Talman and W. F. Shadwick, Phys. Rev. A 14, 36 (1976).

[45] D. Koller, F. Tran, and P. Blaha, Phys. Rev. B 85, 155109 (2012).

[46] H. Jiang, R. I. Gomez-Abal, X. Li, C. Meisenbichler, C. A Ambrosch-Draxl, and M. Scheffler, Comput. Phys. Commun. 184, 348 (2013).

[47] M. Shishkin and G. Kresse, Phys. Rev. B 75, 235102 (2007).

[48] L. Schimka, J. Harl, and G. Kresse, J. Chem. Phys. 134, 024116 (2011).

[49] A. F. Fix, F. U. Abuova, R. I. Eglitis, E. A. Kotomin, and A. T. Akilbekov, Phys. Scr. 86, 035304 (2012).

[50] R. Laskowski, N. E. Christensen, P. Blaha, and B. Palanivel, Phys. Rev. B 79, 165209 (2009).

[51] R. Laskowski and P. Blaha, Phys. Rev. B 82, 205104 (2010).

[52] T. Inui and Y. Uemura, Prog. Theor. Phys. 5, 252 (1950).

[53] J. N. Murrell and J. Tennyson, Chem. Phys. Lett. 69, 212 (1980).

[54] A. Y. S. Kung, A. B. Kunz, and J. M. Vail, Phys. Rev. B 26, 3352 (1982).

[55] R. Pandey, M. Seel, and A. B. Kunz, Phys. Rev. B 41, 7955 (1990).

[56] R. Bader and J. Platts, J. Chem. Phys. 107, 8545 (1997).

[57] A. Leitão, R. Capaz, N. Vugman, and C. Bielschowsky, J. Mol. Struct. (THEOCHEM) 580, 65 (2002).

[58] D. Domínguez-Ariza, C. Sousa, F. Illas, D. Ricci, and G. Pacchioni, Phys. Rev. B 68, 054101 (2003). 
[59] J. Carrasco, C. Sousa, F. Illas, P. V. Sushko, and A. L. Shluger, J. Chem. Phys. 125, 074710 (2006).

[60] S. Huzinaga, L. Seijo, Z. Barandiarán, and M. Klobukowski, J. Chem. Phys. 86, 2132 (1987).

[61] L. Seijo and Z. Barandiarán, The Ab Initio Model Potential Method: A Common Strategy for Effective Core Potential and Embedded Cluster Calculations, in Computational Chemistry: Reviews of Current Trends (World Scientific, Singapore, 1999), Chap. 2, pp. 55-152.

[62] H. M. Evjen, Phys. Rev. 39, 675 (1932).

[63] T. H. Dunning, J. Chem. Phys. 90, 1007 (1989).

[64] J. Adachi and N. Kosugi, B. Chem. Soc. Jpn. 66, 3314 (1993).

[65] E. Miyoshi, Y. Miyake, S. Katsuki, and Y. Sakai, J. Mol. Struct. (THEOCHEM) 451, 81 (1998).

[66] F. Aquilante, L. D. Vico, N. Ferré, G. Ghigo, P.-Å. Malmqvist, P. Neogrády, T. Pedersen, M. Pitonak, M. Reiher, B. Roos,
L. Serrano-Andrs, M. Urban, V. Veryazov, and R. Lindh, J. Comput. Chem. 31, 224 (2010).

[67] D. G. Truhlar, Chem. Phys. Lett. 294, 45 (1998).

[68] P. L. Fast, M. L. Sanchez, and D. G. Truhlar, J. Chem. Phys. 111, 2921 (1999).

[69] A. J. C. Varandas, J. Chem. Phys. 113, 8880 (2000).

[70] M. Nisoli, S. De Silvestri, O. Svelto, R. Scholz, R. Fanciulli, V. Pellegrini, F. Beltram, and F. Bassani, Phys. Rev. Lett. 77, 3463 (1996).

[71] H. Bilz and W. Kress, Phonon Dispersion Relations in Insulators (Springer-Verlag, Berlin, 1979).

[72] A. Schüller, S. Wethekam, D. Blauth, H. Winter, F. Aigner, N. Simonović, B. Solleder, J. Burgdörfer, and L. Wirtz, Phys. Rev. A 82, 062902 (2010).

[73] G. A. Russell and C. C. Klick, Phys. Rev. 101, 1473 (1956).

[74] R. P. Feynman, Phys. Rev. 97, 660 (1955).

[75] H. Fröhlich, Adv. Phys. 3, 325 (1954). 